\title{
Ifosfamide Encephalopathy in A 4yr Old with Ewing's Sarcoma
}

\author{
Edwin Dias* and Anusha Dias \\ Professor and Head, Department of Pediatrics, SIMS \& RC, India \\ *Corresponding author: Edwin Dias, Professor and Head, Department of Pediatrics, SIMS \& RC, Mangalore, India
}

\begin{abstract}
Ifosfamide encephalopathy is a fatal toxicity of Ifosfamide therapy. 13 cases have been reported till the year of 2018 worldwide in children and none among Ewing's sarcoma cases. Here we are discussing a unique case of Ifosfamide encephalopathy during the course of treatment of Ewing's sarcoma and was on Ifosfamide therapy and developed encephalopathy. Child improved symptomatically with MESNA and mannitol. Child was found to be normal on follow up and chemotherapy treatment was continued.
\end{abstract}

Keywords: Encephalopathy; Ewing's Sarcoma; Ifosfamide; Mesna

\section{Introduction}

Encephalopathy is a potentially fatal toxicity of Ifosfamide. Clinical manifestation of encephalopathy range from fatigue and confusion to coma and death. Early identification of this toxicity and prompt cessation of Ifosfamide are the essential elements in the management of Ifosfamide encephalopathy. Neurotoxicity has been reported in about $5 \%$ children treated with Ifosfamide for tumour not involving the CNS [1]. The entity of Ifosfamide neurotoxicity can be of different degree, from very light and transient to fatal. Neurotoxicity occurring during or immediately after Ifosfamide infusion were recorded in children with both solid tumours or leukemia. The drug was administered in different chemotherapeutic association and dosage. Concomitant clinical conditions possibly playing a role as risk factors were the administration of other neurotoxic drugs, the presence of cerebral metastasis, a subclinical lysis syndrome, and altered respiratory function. Symptoms were transient and consisted in most cases, but some had partial or generalised seizures. In some cases, the treatment was continued substituting Ifosfamide with cyclophosphamide. Particularly in patients presenting risk factors, attention has to be paid to the risk of Ifosfamide neurotoxicity and rapidly suspend the drug administration to avoid irreparable damage to the CNS [2-8].
Thereafter the treatment can be reassessed. If Ifosfamide is considered the best option for the given case, it could be safely re-administered in association with methylene blue or thiamine. If encephalopathy re-appears, substitution of Ifosfamide with cyclophosphamide could offer the same oppurtunities of cure of the patient. Ifosfamide is an oxazaphosphorine antienoplastic agent (a nitrogen mustard derivative) and is a structural analog of cyclophosphamide [5,9]. Ifosfamide is a prodrug that requires hepatic activation to its cytotoxic metabolite, ifosfamide mustard $[9,10]$. The latter is metabolized by cytochrome $\mathrm{p} 450$ to generate the active alkylating agents, 4-hydroxy-ifosfamide and isophosphoramide mustard [10]. Ifosfamide and its metabolites can penetrate the blood brain barrier, with CNS toxicity occurring in $10-40 \%$ of patients receiving high doses of the drug [4-8]. The exact pathophysiological mechanisms responsible for the development of ifosfamide-induced encephalopathy are unclear, but one of the causes is thought to be the dechloroethylation of Ifosfamide to form chloroacetaldehyde (CAA) [10-12]. The neurotoxic effects of CAA maybe due to
a. A direct neurotoxic effect,
b. Depletion from the CNS of Glutathion, and/or 
c. The inhibition of mitochondrial oxidative phosphorylation resulting in impaired fatty acid metabolism.

\section{Case Summary}

A 4 yr old, male child presented with $\mathrm{H} / \mathrm{O}$ swelling of right arm noticed in 1st week of march. Father has developed a bone tumour about 3 years back and was on treatment. O/E: Ht: $94 \mathrm{cms}$, Wt:12.1 Kgs, Pulse: 100/min, RR: 24/min, BP:100/70mm Hg Initial Systemic examination: WNL, Local examination: tenderness around right humerus and elbow, with bony swelling of right humerus and right elbow. INVESTIGATION: RFT, LFT: WNL, Ca:9.2P:4.5LDH:863, PS: mild neutrophilia Hb: 11.5gm\%, TC:12900cells/mm. Plain X-ray: Right humerus with shoulder and elbow joint. Radiological features: Signs of Ewing's Sarcoma, Chest X-ray: W.N.L, no evidence of metastasis [13-15].

\section{Biopsy Diagnosis}

Showing bony speckles with a neoplastic infiltrate in the marrow cavity. Infiltrate is composed of cohesive sheets of small round cells with hyperchromic round nuclei. Interstitium have mild fibrosis. Fragments of necrotic bone are also seen. Peripheral reactive new bone formation is also seen, suggestive of Ewing's Sarcoma. The child was put on the following Chemotherapy: Inj. Ifosfamide $1.6 \mathrm{~g} / \mathrm{m} 2$ dissolve in $540 \mathrm{ml}$ N.S. over 3 hours, Inj. MESNA $400 \mathrm{mg} / \mathrm{m} 2 /$ dose-3doses at $3 \mathrm{hr}$ interval after Ifosfamide, Inj. Etoposide $100 \mathrm{mg} / \mathrm{m} 2$ in $540 \mathrm{ml}$ N.S. over 1 hour, 2 weeks later child developed fever with convulsions and oropharyngeal Bacterial and candidly infection with a patch [16]. Child has altered sensorium, had a right UMN facial palsy and right ear discharge. the child was investigated ,Hb: 7.8g\%,TC: 5900cells/ mm3,N: 44\%,L:54\%,E:2\%,ESR: 90mm/hour, RFT: W.N.L.S. electrolytes: W.N.L., Urine: 3-4 WBCs, Protein: +,Throat swab: Gram stain: Gram-ve bacilli and Gram +ve cocci are seen Culture and sensitivity report: Pseudomonas spp and Staphylococci have grown. No fungus has grown. CT chest and right humerus and right elbow: no evidence of metastasis, EEG was suggestive of metabolic encephalopathy. Right Ewing's sarcoma of humerus and elbow. Ear swab: Pseudomonas saprophyticus resistant to first line antibiotics. Meningitis and CNS secondaries were ruled out and a diagnosis of Ifosfamide induced encephalopathy was made. Child improved with Mannitol, Anticonvulsants, Antibiotics and antifungals. Child started on alternative chemotherapy (CUA regimen) and was found to be normal on follow-up.

\section{Discussion}

Child had presented with c/o swelling in the right arm and was diagnosed to have Ewing's sarcoma, which was confirmed on biopsy. Child was started on Inj Ifosfamide, Inj Mesna and Inj Etoposide. 2 weeks later child developed fever with convulsions and oropharyngeal Bacterial or candidial infection with a patch. Child has altered sensorium. Had a right UMN facial palsy and ear discharge. A diagnosis of Ifosfamide induced encephalopathy was made. Child improved with mannitol, anticonvulsants, antibiotics and antifungals. CNS toxicity of Ifosfamide displays a wide spectrum of signs and symptoms. Manifestations of Ifosfamide induced encephalopathy include cerebellar ataxia, mental confusion, complex visual hallucinations, extrapyramidal signs, seizures and/or mutism. Less common manifestations include asterixis, non-convulsive status epilepticus, manic episodes and cerebellar and temperofrontal cortical degeneration. Ifosfamide induced encephalopathy is a clinical diagnosis [17]. The differential diagnoses of Ifosfamide induced encephalopathy include infection, metabolic abnormalities and concomitant drug induced syndromes. It is supported by normal brain imaging, EEG findings of metabolic encephalopathy and the absence of other causes. EEG characteristics have been correlated with the clinical grading of Ifosfamide induced encephalopathy. Methylene blue was found to be useful in managing cases of Ifosfamide induced encephalopathy

\section{References}

1. Di Cataldo A, Astuto M, Rizzo G, Bertuna G, Russo G, et al. (2009) Neurotoxicity during Ifosfamide treatment in children; et al. Med Sci Monit 15(1): CS22-CS25.

2. Shin YJ, Kim JY, Moon JW, You RM, Park JY, et al. (2011) Fatal IfosfamideInduced Metabolic Encephalopathy in patients with recurrent Epithelial Ovarian Cancer: Report of two cases. Cancer Res Treat 43(3): 260-263.

3. Giovanis P, Garna A, Marcante M, Nardi K, Giusto M (2009) Ifosfamide encephalopathy and use of methylene blue. A case report of different sequential neurotoxicity. Tumori 95(4): 545-546.

4. Ajithkumar T, Parkinson C, Shamshad F, Murray P (2007) Ifosfamide encephalopathy. Clin Oncol (R Coll Radiol) 19(2): 108-114.

5. Alici Evcimen Y, Breitbart WS (2007) Ifosfamide neuropsychiatric toxicity in patients with cancer. Psych oncology 19(10): 108-114.

6. Rieger C, Fiegl M, Tischer J, Ostermann H, Schiel X (2004) Incidence and severity of Ifosfamide-induced encephalopathy. Anticancer Drugs 15(4): 347-350.

7. Meanwell CA, Blake AE, Kelly KA, Honigsberger L, Blackledge G (1986) Prediction of Ifosfamide/MESNA associated encephalopathy Eur J Cancer Clin Oncol. 22(7): 815-819.

8. Klastersky J (1999) Side effects of Ifosfamide-induced neurotoxicity. Pharmacotherapy 19: 1450-1455.

9. McVay JI, Wood AM (1999) Suspected Ifosfamide induced neurotoxicity. Pharmacotherapy 19(12): 1450-1455.

10. Pelgrims J, De Vos F, Van Den Brande J, Schrijvers D, Prové A, et al. (2000) Methylene Blue in the treatment and prevention of Ifosfamide-induced encephalopathy: report of 12 cases and a review of the literature. Br J Cancer 82(2): 291-294.

11. David KA, Picus J (2005) Evaluating risk factors for the development of ifosfamide encephalopathy. Am J Clin Oncol 28(3): 277-280.

12. Patel PN (2006) Methylene blue for management of Ifosfamide-induced encephalopathy. Ann Pharmacother 40(2): 299-303.

13. Küpfer A, Aeschlimann C, Cerny T (1996) Methylene blue and the neurotoxic mechanisms of ifosfamide encephalopathy. Eur J Clin Pharmacol 50(4): 249-252.

14. Turner AR, Duong CD, Good DJ (2003) Methylene blue for the treatment and prophylaxis of ifosfamide-induced encephalopathy. Clin Oncol (R Coll Radiol) 15(7): 435-439. 
15. Donegan S (2001) Novel treatment for the management of ifosfamide neurotoxicity: rationale for the use of methylene blue. J Oncol Pharm Pract 6(4):153-165.

16. National Cancer Institute (2003) Common Terminology Criteria for Adverse Events (CTCAE) version 3.0. Bethesda, MD: National Cancer Institute.
17. Brunello A, Basso U, Rossi E, Stefani M, Ghiotto C, et al. (2007) Ifosfamiderelated encephalopathy in elderly patients: report of five cases and review of the literature. Drugs Aging 24(11): 967-973.

\section{(C) (P) \\ This work is licensed under Creative Commons Attribution 4.0 License}

To Submit Your Article Click Here:

Submit Article

DOI: 10.32474/PAPN.2019.02.000133

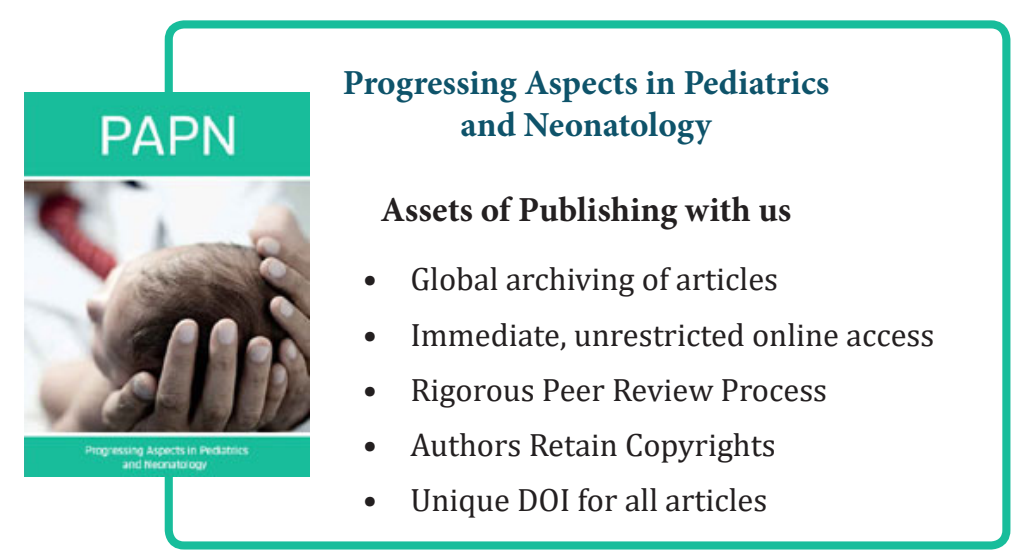

